vinism, there is little hope that the causes of disasters will be addressed, and it is likely that, no matter what technical progress is made, relief activities will remain stopgap efforts that treat the only symptoms.

Keywords: coordination; Geneva Conventions; human rights; humanitarianism; international disaster response law; international humanitarian law; International Red Cross; post-traumatic stress disorder (PTSD); relief; standards; terrorism

Prehosp Disast Med 2002;17:s1-2.

\section{Disaster Management Evaluation: The Current Status of the Standardised Protocol in the Utstein Style KO Sundnes; ML Birnbaum}

Task Force on Quality Control of Disaster Management (TFQCDM), Norway

University of Wisconsin, Madison, Wisconsin USA

Introduction: For decades, disasters and events that result in multiple casualties have provoked humanitarian responses to assist the affected population regardless of whether they were caused by deliberate, man-made or natural events. However, the statements of the UN Secretary General in $1971,{ }^{1}$ the US Council on Foreign Affairs in 1987,1 and conclusions reached following the hurricanes George and Mitch of the Pan-American Health Organisations (PAHO) ${ }^{2}$ differ little with regard to the overall criticism of how international disaster management functions. Lack of such improvement can be attributed to the absence of formalized and appropriate research methods.

Purpose: The Guidelines and Templates presented in this document propose a structural framework to be used to appropriately design, conduct, and report findings of evaluation and research in Medical and Public Health aspects associated with disasters. Studies performed using this structure will provide the ability to compare and integrate the findings of the evaluations and research of different disasters with the end-point to attenuate or eliminate disasters. This could result from the elimination or reduction of hazards, decreasing the risks for the actuation of the hazard, augmenting the absorbing capacity of the society and environment at risk to prevent the hazard from becoming a destructive event, and enhancing the efficiency, effectiveness, and cost:benefit of preparedness and responses to disasters.

The Guidelines: This report is the result of extensive research and discussions by a Steering Committee over a course of seven years, and by invited participants in two International Workshop (Gothenburg, Sweden, 1997 and Lyon, France, 2001). The process was endorsed by the General Assembly of the World Association for Disaster and Emergency Medicine (WADEM) in Osaka in 1999. ${ }^{3}$ Since summer 2000, the World Health Organisation (Department for Emergency and Humanitarian Action) has participated both in the ongoing development and in its implementation as basis for research and teaching.

The major impediments that influence the conduct of research and evaluations of disasters have been addressed systematically as the four pillars of importance to support the "Table of Research":

1. Conceptual Framework comprises standardized definitions and concepts necessary to minimize confusion;

2. Scientific Methods comprise methods validated by the social sciences and applied to disaster research and evaluation;

3. A Template identifies chronological phases and functions that should be incorporated into the structure of research and evaluation projects. (Regardless of type of disaster: the phase of impact may vary from seconds (earthquakes) to years (droughts), but still is the same phase.)

4. Inventory comprises a list of the Basic Societal Functions as well as the potentially appropriate indicators of change from pre-event baselines to be followed through the disaster phases.

This permits comparative research with both internal and external validity. In this context, the concept of "Best Outcome Without Assistance (BOWA) will be developed. ${ }^{3}$ The ultimate goal is to identify and modify these key factors causing disasters before they happen. The conceptual framework allows such analysis. Complex emergencies and terrorist acts are included in this objective.

Appropriate Indicators will be identified to measure and differentiate between efficiency, effectiveness, and benefit. One commodity (or action) may serve both as a independent and dependent variable. Indicators may be quantitative or qualitative. Those indicators required for computation of the Disaster Severity Score and Health Disaster Severity Score have been developed. Further indicators must be developed during further use of the guideliens.

Conclusion: Use of the Disaster Research Template should provide all elements needed to analyse disasters, their potential for reduction, and their management in an institutionalised manner.

References:

1. HRH Prince Sadruddin Aga Khan: Improving Disaster Management of the United Nations. United Nations Management \& Decision-making Project, UNA-US, New York

2. PAHO/WHO: Evaluation of preparedness and response to Hurricanes George and Mitch: Conclusions and recommendations. Prehosp Disast Med 1999:14;53-65.

3. TFQCDM, Chair: Sundnes KO: Health disaster management: Guidelines for evaluation and research in the Utstein style: Executive summary. Prebosp Disast Med 1999:14;43-52.

Keywords: basic elements; BOWA; damage probability; disaster medicine; evaluation; qualitative methods; quantitative methods; research; severity scores; template; vulnerability

Prehosp Disast Med 2002;17:s2

\section{Future of Disaster Medicine}

Disaster Medicine in the 21st Century

Jeffrey Arnold, MD, FACEP, FAAEM

Department of Emergency Medicine, Baystate Medical Center, Tufts University School of Medicine, Springfield, Massachusetts USA

The events in New York City and Washington, DC on 11 September, 2001 not only marked the largest, single, coordinated terrorist attack in history, but the resulting disinte- 